\title{
Sciatic pain after rubber band ligation of haemorrhoids
}

\author{
Avi Levin • Joseph Lysy
}

Accepted: 28 September 2010/Published online: 19 October 2010

(C) Springer-Verlag 2010

\section{Dear Editor:}

Rubber band ligation is probably the most commonly used nonsurgical treatment for haemorrhoidal disease. Tissue destruction by heat delivered in a variety of forms is slightly less effective (needs more re-treatment) but is significantly less painful. During the last 10 years, more than 1,000 patients had rubber band ligation of haemorrhoids in our proctology clinic. Here, we report a 30 -year-old female who developed severe sciatic pain and paresthesis of the left leg immediately after rubber band ligation of the left lateral internal haemorrhoids. The pain was severe and localised to the left buttock, radiating through the back of the left thigh down to the ankle. Surprisingly, the anal pain was minimal. Physical examination was unremarkable. The location of the rubber band was $10 \mathrm{~mm}$ above the dentate line. Two hours later, the ring was removed due to intolerable "sciatic like pain" and paresthesis. Following the rubber band removal, the pain was immediately alleviated and completely resolved within a few minutes.

We do not understand the exact pathogenesis of the phenomenon. Upper anal canal is insensitive to stimuli-like cutting or squeezing, allowing us to band haemorrhoids without anaesthesia. On the other hand, stimuli-like tension and traction are conducted by the sensory nerves, producing visceral dull pain. These sensory fibres run in the parasympathetic nerves and enter the spinal cord at the same segments (S2-S4) like a part of the sciatic nerve, innervating to the buttock area. Due to the proximity of those nerves, we can speculate that impulse delivered by one could be interpreted as delivered by another. The other option is that local inflammatory reaction to rubber band ligation can trigger adjacent nerves and create false sensation as we see sometimes in an infection of the pelvic floor. Interestingly, four out of five patients, reported in literature with this complication, were females, raising the possibility that female pelvic anatomy could be a predisposing factor to this complication.

Sciatic-like pain caused by rubber band ligation is extremely rare, and to the best of our knowledge, this is the second report of such a complication. In the previously reported cases, the rubber band was left in place, and the duration of the "sciatic like pain" was 7 days. We preferred to release the rubber band, which resulted in prompt pain resolution, and to refer the patient to argon plasma laser haemorrhoidal treatment. 